\title{
Mythbusters! The Truth about Common Misconceptions in Cochlear Implantation
}

\author{
Erika A. Woodson, M.D., ${ }^{1}$ Ksenia Aaron, M.D., ${ }^{1}$ \\ Ahn Nguyen-Huynh, M.D., Ph.D., ${ }^{1}$ Jonathan Vargo, M.D., ${ }^{2}$ and \\ Sarah E. Mowry, M.D.2,3
}

ABSTRACT

Cochlear implantation (CI) is the preferred method of hearing rehabilitation when patients cannot perform well with traditional amplification. Unfortunately, there are still significant misconceptions around this life-changing intervention. The goal of this article is to address some of the most common myths around CI surgery. After reading this article, the learner will be able to explain the utility of $\mathrm{CI}$ in patients with residual hearing and recognize that insurance coverage is widespread. The reader will be able to list common risks associated with this well-tolerated procedure including anesthetic risk and the risk of vestibular dysfunction. Additionally, the reader will be able to identify the significant positive impact of CI on patients' quality of life. Finally, the reader will identify that many patients can safely have an MRI scan after implantation, including nearly all contemporary recipients.

KEYWORDS: cochlear implants, outcomes, hearing loss, vestibular dysfunction, insurance, hearing preservation, MRI compatibility, anesthesia

Cochlear implantation (CI) has become a well-established (re)habilitation option for patients with moderate to profound hearing loss who do not benefit from traditional amplification. After more than 30 years of experience using this technology for both adults and children, the benefits for recipients are significant and well understood. Despite their proven track record for improving both audibility and speech understanding, there are still misconceptions about the device itself, perioperative management, and postoperative outcomes. The goal of this article is to dispel common myths and provide the reader with evidence to support the truth about CI.

Myth: Patients with residual low-frequency (LF) hearing do not benefit from CI.
${ }^{1}$ Head and Neck Institute, Cleveland Clinic Foundation, Cleveland, Ohio; ${ }^{2}$ Department of Otolaryngology-Head and Neck Surgery, Case Western Reserve University School of Medicine, Cleveland, Ohio; ${ }^{3}$ Department of Otolaryngology-Head and Neck Surgery, University Hospitals Cleveland Medical Center, Cleveland, Ohio.

Address for correspondence: Sarah Mowry, M.D., 11100 Euclid Avenue, LKS5045, Cleveland, OH 44106 (e-mail: Sarah.Mowry@UHHospitals.org).
Barriers to Cochlear Implant Access: Acknowledging the Challenges, Changing the Future; Guest Editor, Sarah Sydlowski, Au.D., Ph.D., M.B.A.

Semin Hear 2021;42:352-364. (C) 2021. Thieme. All rights reserved. Thieme Medical Publishers, Inc., 333 Seventh Avenue, 18th Floor, New York, NY 10001, USA DOI: https://doi.org/10.1055/s-0041-1739368.

ISSN 0734-0451. 
Fact: Earlier implantation when LF hearing is still serviceable allows a patient to enjoy tangible benefits of acoustic and implant hearing together-electroacoustic stimulation (EAS).

Hearing preservation (HP) is one of the most critical advances in modern CI surgery. Through advances in technique and technology, patients now can access the benefits of CI earlier, before being severely hearing impaired through all frequencies. Traditional criteria for CI allowed only severe-to-profound sensorineural hearing loss (SNHL) to benefit from the technology. ${ }^{1}$ Because of concerns of residual hearing loss, individuals with partial SNHL were excluded from implantation initially. Individuals with serviceable LF thresholds remained outside of traditional CI criteria, even if they had profound high-frequency SNHL and poor speech discrimination. Later, published series of patients proved that measurable hearing after CI was feasible. ${ }^{2,3}$ Although the initial studies reported preserved hearing which was measurable and not serviceable, these early reports cemented the proof of concept that complete acoustic hearing loss after CI was not a certainty.

Several surgeons challenged the conventional wisdom that CI would only help patients with profound, and not partial, SNHL. In the United States, Gantz and Turner developed a series of short, thin partial length electrode arrays, the Cochlear Iowa/Hybrid CIs (Denver, $\mathrm{CO}$ ), starting in $1999 .{ }^{4}$ Initial HP results were quite good with $90 \%$ subjects preserving functional HP immediately postoperative, and $80 \%$ of patients maintaining functional $\mathrm{HP}$ at the end of the trial, 2 years out from surgery. ${ }^{5}$ Notably patients did not benefit as greatly from this shortest prototype, the 6-electrode, $10 \mathrm{~mm} \mathrm{~S} 8$ (65\% CNC words at 6 months postactivation) as the later longer prototypes S12 and L24 (81 and 83\% at 6 months postactivation, respectively). ${ }^{6}$ Ultimately, the FDA cleared the Cochlear Hybrid L24 implant in 2014. ${ }^{7}$ Concurrently in Europe, enthusiasm for thinner, shorter cochlea implant arrays resulted in the development of the MED-EL Flex+ 24 (Innsbruck, Austria) electrode array, which was FDA approved in 2012. ${ }^{8}$

Importantly, patients could be offered the Hybrid L24 or FLEX + EAS implants with vastly more inclusive criteria than traditional CI. EAS criteria for both arrays allow for good to normal ( $<60 \mathrm{~dB}$ and $<65 \mathrm{~dB}$ for Hybrid L24 and FLEX +24 , respectively) LF hearing, and $<60 \%$ CNC Word recognition in the ear to be implanted on aided testing. ${ }^{7,8}$

Gantz et al's work demonstrated that patients did better with their CI than they did preoperatively. ${ }^{6}$ Moreover, their patients received their optimal benefit when the LF acoustic hearing was preserved and utilized. ${ }^{9}$ If a patient had postoperative SNHL, they were fitted with an acoustic hearing aid integrated into the CI's speech processor, an EAS system.

Preservation of LF hearing means little if it is not serviceable or if it remains unaided after CI activation. The acoustic component works like a traditional hearing aid, often with a receiver-incanal microphone and amplifier. The acoustic component delivers amplification to the residual LF acoustic thresholds, while the processor delivers the mid and high frequencies through the CI. The unit is programmed so that the cut-offs for acoustic delivery and electrical delivery are optimized, usually with some overlap, and a full range of sound detection is achieved. ${ }^{10}$

Benefits to the retention and amplification of LF hearing are multiple. Most importantly, individuals perform better in background noise. ${ }^{11}$ Squelch is a cortical phenomenon requiring even, bilateral auditory input. It relies on spectral and temporal cues that then allow higher-level processing to focus listening on a single sound, namely conversation, out of a complex listening background, that is, background noise. The temporal and spectral cues received through a CI are different than with acoustic hearing, which affects the brain's ability to apply them together to attain the same result in background noise. The retention of LF hearing allows for some acoustic information to be received and processed bilaterally-which is critical for squelch to occur effectively.

Other tangible benefits to preserved LF hearing are better sound localization and superior music appreciation. ${ }^{12}$ Like squelch, sound localization is also a cortical phenomenon requiring bilateral auditory input with the same spectral and temporal cues. Although patients in the bimodal condition (CI in one ear and hearing aid in the other ear) perform better than 
patients with unilateral input, it is not as accurate as when both the two ears can process acoustic information symmetrically and simultaneously. ${ }^{13,14}$

Improved music appreciation was noted during the long-term follow-up of Hybrid trial patients. Parkinson et al demonstrated that LF acoustic information was vital for discerning melody. ${ }^{15}$ Without LF hearing, CI-only listening condition limited patients to rhythm recognition of novel songs, and appreciation of familiar melodies from the subjects' pre-deafened past. ${ }^{16}$

Numerous case series of HP outcomes after traditional surgery have been published. The average patient will experience a threshold shift of approximately $20 \mathrm{~dB}$ after soft surgery with modern slim arrays. ${ }^{17}$ Additionally, delayed loss of HP may occur over time, any time after activation. In the study by Mamelle et al, a large series of patients implanted with various slim arrays were noted to have a cumulative risk of $50 \%$ total $\mathrm{HL}$ at 7 years postoperative. ${ }^{18}$ If delayed HL occurs, these threshold shifts will adversely affect programming. Upon the identification of any shifts in the acoustic hearing, a different allocation MAP must be provided to incorporate those lost frequencies into the speech processor so that a full range of sound information can continue to be received.

Although measurable acoustic hearing can frequently be achieved, serviceable hearing may not. To standardize reporting in scientific journals, the American Academy of Otolaryngology-Head and Neck Surgery Hearing Committee created a reporting guideline for articles investigating HP after CI. ${ }^{19}$ The reporting guideline set a standard LF $(125,250$, and $500 \mathrm{~Hz})$ PTA of $\angle 80 \mathrm{~dB}$ as the threshold of success. Technically, an acoustic hearing aid could be programmed to fit LF postoperative acoustic thresholds approaching $80 \mathrm{~dB}$, but many patients do not perceive benefit to wearing an acoustic component at these minimal hearing levels. ${ }^{20}$ If significant threshold shifts occur postoperatively, or if preoperative thresholds are not mild/mild-moderate, the patient may not benefit from or opt to wear an acoustic component postoperatively. Importantly, even if serviceable/measurable hearing is lost, the benefits to speech recognition in the CI-only condition are undeniable. Even in circumstances of profound postoperative acoustic hearing, patients perform much better post-CI than pre$\mathrm{CI}$, and rate their satisfaction with their $\mathrm{CI}$ the same. ${ }^{21}$

The new FDA indications for these partial length arrays opened a new window of opportunity to patients living with partial hearing loss. Concurrent with the refinement of the Hybrid, each of the CI manufacturers on the U.S. market developed less traumatic electrode arrays that could potentially spare LF residual hearing and allow for EAS: the Cochlear Nucleus 622, 632, the MED-EL FLEX+ 28, and the Advanced Bionics (Valencia, CA) HiFocus MidScala and Slim J. However, the aforementioned electrode arrays carry the FDA labeling of traditional CI candidacy, not EAS specifically. Nonetheless, these arrays can be deployed when a patient has any serviceable LF hearing because each of the FDA-approved CI manufacturers features an external processor with an optional acoustic component to enable EAS.

In summary, the ability to preserve and use LF acoustic hearing has effected great change on who is currently considered a CI candidate. $\mathrm{CI}$ is offered earlier, before all thresholds drop to severe-profound levels. Overall, the benefits of CI greatly outweigh the risk of residual hearing loss at ear-specific performance levels when a patient meets CI criteria based on sentence (traditional) and/or word (EAS) recognition scores. When appropriate, an acoustic component to amplify the residual hearing further enriches a patient's sound environment and improves the outcome measures which rely on higher-level processing of bilateral sound input.

Myth: Insurance will not cover CI.

False: The vast majority of insurance carriers and plans cover the perioperative and (re) habilitative costs associated with CI surgery.

Although many insurance providers, including Medicare, do not cover traditional hearing aids, CI is a medically covered benefit by most insurance plans in the United States. According to the American Cochlear Implant Alliance, an advocacy group for CI access, 90\% of private or employer-sponsored plans explicitly cover costs associated with $\mathrm{CI}{ }^{22}$ Federal 
legislation has been introduced at the time of this publication to mandate coverage of CI by private insurance plans (Ally's Act, HR 477). ${ }^{23}$ Medicare Part B also covers $80 \%$ of the costs associated with implantation of one ear for those who meet Medicare's audiometric criteria. Commercial supplemental plans generally cover most of the $20 \%$ that Medicare does not pay, minus the plan's co-pay/co-insurance cost. These carriers may require preauthorization of services while Medicare Part B does not. Similarly, Medicare Advantage plans typically offer coverage for CI, but these plans may also require preauthorization of services. Both Medicare Advantage and commercial plans may offer flexibility to recommend CI off-label, while Medicare Part B does not allow off-label coverage.

CI may be covered by publicly available plans available through the federally mandated health insurance exchanges. If the essential health benefit plan in a given state explicitly covers $\mathrm{CI}$, then all other plans on the exchange provide $\mathrm{CI}$ as a benefit. However, inclusive language covering $\mathrm{CI}$ is not always present. In these cases, CI may be covered under habilitative/rehabilitative services or even under durable medical equipment. If there is a question about coverage of CI by a specific plan, patients are encouraged to contact their insurance carrier.

Medicaid coverage for children younger than 21 years is covered by all state Medicaid plans as a habilitative service under federal statue. However, for adults, Medicaid coverage is highly variable. Unfortunately, while the states are required to provide coverage for children, reimbursement of these procedures to the hospitals and other providers continues to be low; in some states, the reimbursement is so low that many private hospitals or providers cannot offer the service.

The Veteran's Health Administration and TriCare also cover CI. VA eligible veterans with service connection for hearing loss and those with more than $10 \%$ overall service connection are eligible for full coverage of all costs associated with CI. Not all VA centers offer CI surgery and patients may be required to travel to the CI center in their regional VA network (VISN) for surgery. In many VISNs, audiological services can be provided at a center closer to where the Veteran lives and receives their routine care.

Costs associated with $\mathrm{CI}$ are more than just the surgery itself. Patients have both preoperative and postoperative appointments, and equipment needs. The preoperative evaluation is generally a covered benefit for patients and includes medical/surgical evaluations, radiologic imaging procedures, speech evaluations, audiometric testing, vaccination against pneumococcal disease, and, for children, evaluations by developmental pediatrics and a geneticist. Patients are generally responsible for any co-pays and deductibles associated with these appointments. Genetic testing coverage is highly variable and is not covered under many Medicaid plans.

Postoperative support for patients includes continued audiologic follow-up, hardware repairs and replacement, batteries (for those without rechargeable batteries), and intensive speech and language therapy. Insurance coverage for the external hardware is generally covered under medical necessity but may require documentation from the surgeon. Habilitative services, including speech language therapy, for children are also a covered benefit. However, the number of sessions per year may be restrictive and jeopardize continued improvement for children needing intensive speech and language therapy. Often therapy providers and families can successfully request additional sessions with the appropriate documentation.

In summary, the process of qualifying for $\mathrm{CI}$, the surgery itself, and the necessary aftercare are covered benefits for most Americans. Patients and providers are encouraged to check with their insurance carrier to determine the specific coverages of their plan.

Myth: CI surgery is a dangerous/high-risk operation.

Fact: CI surgery is safe and well tolerated. Nonetheless, there are risks associated with surgery and the implant itself that the patient must understand before undergoing the procedure.

\section{Risk of Anesthesia}

Multiple factors go into the evaluation of the patient and determination of CI candidacy. 
Prior to surgery, it is essential to ensure that appropriate physical examination, history, imaging, and audiological and preoperative medical risk stratification examinations are performed, ensuring safe and appropriate surgical management. Careful evaluation of the overall health of an adult patient, especially in the elderly category, is essential as many patients have multiple comorbidities and medications that play a role in increasing the risk of general anesthesia. Anesthesia, in otherwise healthy adults, is generally safe. Total intravenous anesthesia is used during most $\mathrm{CI}$ cases to allow for facial nerve monitoring.

In the elderly population, postoperative delirium, usually a temporary condition, and postoperative cognitive dysfunction, which can lead to long-term memory loss and make it difficult to concentrate, have been described. ${ }^{24}$ Predisposing conditions, including heart, lung, and vascular disease, as well as a history of a stroke in the past, increase the patient's risk for these adverse outcomes. ${ }^{24}$ Several studies looking specifically at risk assessment of age and anesthesia in CI recipients have had various outcomes. Some found that advanced age did not play a role as an independent risk factor for general anesthesia but rather an overall physical status and medical well-being of the patient. ${ }^{25}$ However, another study showed that older CI recipients, older than 80 years, were more likely to experience complications due to anesthesia. ${ }^{26}$ Medical comorbidities such as hypertension (56\%) and heart disease (53\%) are common in patients older than 85 years who receive CI. ${ }^{27}$ Additionally, geriatric patients are more likely, than younger adults, to have cerebrovascular events (4\%) and cardiac arrhythmia (4\%) secondary to anesthesia. Overall, there is a low complication rate with minimal to no longterm morbidity or mortality in older adults undergoing CI surgery, occurring in less than $5 \%$ of patients, indicating that the overall risk of anesthesia is still low in geriatric patients. ${ }^{26-28}$

An alternative to general anesthesia, local anesthesia, or local anesthesia with minimal conscious sedation for patients undergoing CI surgery has been recently described by several institutions. ${ }^{29-32} \mathrm{CI}$ surgery under local anesthesia appears to be a safe and effective alternative for patients who may not tolerate general anesthesia. Some suggest that the use of local anesthesia avoids roughly $9 \%$ mortality as predicted by the preoperative risk-adjusted scoring system. ${ }^{33}$ Other studies reported that CI surgeries under conscious sedation with local anesthesia decreased surgical time and drug costs to the patients with no differences in perioperative morbidity compared with patients implanted under general anesthesia. ${ }^{32}$ An additional study demonstrated that local anesthesia, used for $71 \%$ of study patients older than 85 years, was safe. ${ }^{27}$ This surgical technique, when used with an experienced anesthesiologist, may be an alternative for older adult CI candidates with multiple comorbidities in whom general anesthesia might be contraindicated.

\section{Postoperative Complications}

Surgical risks are divided into two groups, major and minor, with an overall prevalence of complications at $12.8 \%{ }^{34}$ The complication group depends on whether the treatment requires a surgical intervention/hospital admission, or the patient can be managed with an outpatient treatment/observation. Prevalence of major complications for a $\mathrm{CI}$ recipient is reported at $2.7 \%$ and includes device failure, flap dehiscence, meningitis, facial nerve paralysis, and other implant barriers, including migration or extracochlear insertion requiring explantation. ${ }^{34-36}$ Minor complications include vertigo, dizziness or imbalance, facial nerve paresis or stimulation, dysgeusia, and tinnitus. ${ }^{34-36}$

\section{Device Failure}

While device failure is technically not a surgical complication, it is one of the most common indications for revision surgery. There are three categories of device failure: hard failure, soft failure, and medical failure. Hard failures of CI happen when the implant stops working due to device malfunction or structural problem. ${ }^{37}$ Soft failure occurs when there is suspected device problem due to decline in performance, adverse auditory symptoms, shocking sensation, or intermittent malfunction of the device. In these cases, the etiology can only be confirmed by removing and examining the suspect device. ${ }^{37,38}$ The third category of failure is medical 
failure. Medical failure would entail issues such as infection, soft-tissue breakdown, or other medical problems of the implant recipient and not the device itself. A large study, which part of its cohort examined 1,499 hearing-impaired adult CI recipients, reported a $4.1 \%$ failure that necessitated explantation. ${ }^{39}$ Another 30 -year retrospective analysis of 2,827 CI adult and children recipients showed that $8.1 \%$ of patients required revision surgery, and of those, $57.8 \%$ were due to device failure. ${ }^{40}$ The overall prevalence of CI device failures in adults is reported at $1.9 \%$, with reports demonstrating variability between 0.8 and $5.7 \% .^{34,41,42}$ Most of all patients who require device explantation due to failure are eligible to be reimplanted. The success of reimplantation to achieve functional benefit has been reported to decrease with increased age. ${ }^{43}$

\section{Infectious Complications}

Minor infections: Postsurgical infections have drastically declined since the use of antibiotics. CI surgery is generally classified as cleancontaminated due to the risk of pathogenic translocation through the eustachian tube. ${ }^{44}$ $\mathrm{CI}$ is a foreign material, and in some implant recipients, inflammation and erythema can happen. Postoperative infection for CI is estimated to be around $1.9 \%$ in otherwise healthy adults, with higher percentages seen in adults with underlying chronic health conditions. ${ }^{34}$ If an infection is suspected, intravenous antibiotics are started; in some cases, surgery to clear the infection and possible explantation is necessary.

Serious skin complications: Flap necrosis and device exposure can be caused by numerous factors such as local skin infection, shape and thickness of the flap, or other variables. Complications due to flap-related issues, in adults, arise usually because of thinning of the skin with age, as well as underlying conditions including diabetes mellitus or long-standing cardiac, vascular, and renal-related conditions. ${ }^{45}$ Excessively strong magnet can be another contributing factor leading to flap necrosis/infection. ${ }^{46}$ In up to $13 \%$ of elderly CI recipients, flap thinning is observed over long-term follow-up. ${ }^{47}$ Nevertheless, this rarely requires implant removal or flap revision. Flap necrosis or skin breakdown has an average reported rate between 0.3 and $1.4 \% .^{34,48}$ Of those, the primary intervention administered depended on skin complication severity in which revision surgery with wound closure over an implant or revision surgery with explantation is performed. ${ }^{45}$

Meningitis: Meningitis is a life-threatening complication. Until 2002, it was reported that the population with $\mathrm{CI}$, especially children, were at a 30-fold increased risk rate of developing postsurgical meningitis when compared with age-matched peers not implanted. ${ }^{49}$ The risk was associated with the use of an incorporated electrode positioner, which has since been taken off the market. The overall prevalence of meningitis in the postoperative setting is less than $0.1 \%{ }^{34}$ Vaccination for high-risk strains of Streptococcus pneumoniae is mandatory for all implant recipients to minimize the possibility. ${ }^{50}$ During CI surgery, preventing dural exposure during mastoidectomy, atraumatic electrode insertion, and sealing off cochleostomy after a CI with soft tissue must be taken to minimize the risk of meningitis. ${ }^{51-54}$

\section{Facial Nerve Risk}

The facial nerve innervates the facial muscles that stimulate facial movement as well as gives rise to a nerve branch that provides taste sensation of the tongue. Both functions can be potentially altered during CI surgery. Fortunately, both temporary and permanent occurrences of these complications are very infrequent. A study of 705 adult and pediatric patients with $\mathrm{CI}$ demonstrated the incidence of facial nerve paresis to be $0.71 \% .{ }^{55}$ Most studies report a range between 0.1 and $4.8 \% .^{48,56-58}$ In almost all cases, the weakness resolves to normal function with time. To reduce injury to the facial nerve, a facial nerve monitor is used during surgery, and during surgery, drilling of the facial recess is done under continuous irrigation with saline which decreases thermal injury. When delayed facial weakness is observed postsurgically, treatment with a course of corticosteroid taper is recommended. The prevalence of complete facial nerve paralysis has been reported in $0.1 \%$ of cases. ${ }^{34}$ 
Aberrant facial nerve stimulation is another complication that can occur during CI surgery when electrodes are in close proximity to the facial nerve. Reprogramming the processor or selectively deactivating the offending electrode can often resolve this problem. It is not uncommon to have at least one electrode turned off for various reasons, including mapping, adverse symptoms, and others, with reported rates of $54 \%{ }^{42}$ In extremely rare cases, facial stimulation persists despite optimization, and the patient may become a nonuser or even request removal of the internal component. In adults, facial nerve stimulation from a $\mathrm{CI}$ ranges between 0.9 and $15 \%{ }^{59-61}$

Altered taste is another potential complication after $\mathrm{CI}$ implant surgery. It results when the chorda tympani nerve is disturbed during surgery. In most cases, this nerve is preserved; however, thermal damage sustained from the heat of the drill or aberrant stimulation from electrodes can lead to dysfunction. An incidence of transient gustatory changes has been observed in the range of 0.4 to $25 \%$ in CI patients. $57,58,62$ As long as the nerve is preserved, most patients are able to achieve a return to normal taste function within 6 weeks postoperatively.

\section{Tinnitus}

Tinnitus has been reported as a common symptom, seen in up to $80 \%$ of patients prior to CI. ${ }^{63,64}$ Some studies report on the resolution of existing tinnitus after CI surgery in up to $93 \%$ of patients. ${ }^{64}$ Nevertheless, others report that tinnitus persists despite CI in up to $72 \%$ of patients. ${ }^{65}$ Studies assessing the incidence of new tinnitus postimplantation describe a range between 0.2 and $25 \%$ of cases with an overall prevalence of $0.3 \%$ as found in large studies. ${ }^{48,66,67}$

Myth: The CI will sound bad or worse, it will not work for me.

False: The overwhelming majority of patients perceive significant benefit from their CI.

In a meta-analysis by McRackan et al, the authors found significant improvements in patients' quality of life (QOL) which were independent of the objective measures of sound intelligibility. ${ }^{68}$ The areas impacted by CI include communication, listen effort, independence, emotional well-being, ability to participate in entertainment activities, social interactions, and environmental awareness. ${ }^{69}$ The recent validation of a patient-reported outcome measure questionnaire, the CIQOL-35, will expand our understanding further of the impact of CI on patients' experienced quality of life with these devices. ${ }^{70}$ There is also significant improvements in QOL for patients with bilateral CIs beyond speech recognition, as bilateral users have improved task performance for binaural squelch, the head shadow effect, and summation. ${ }^{71-73}$ Extensive preoperative counseling by both programming audiologists and the CI surgeon is imperative to ensure that patients have realistic expectations about what the CI can and cannot do.

Patients often ask what the implant will sound like. The answer to this question depends on a multitude of factors, including, but not limited to, prior hearing status, age, education, and previous sound exposures. CI placement for congenital deafness results in different sound qualities than CI placement for sudden, singleside deafness (SSD) or for patients who are post-lingually deafened. Dorman et al studied comparisons of CI hearing to such sound makers as a vocoder and the like. This showed that CI hearing is closer to speech that has been muffled by band pass filtering and/or spectral smearing. ${ }^{74}$

One of the unique scenarios regarding CI use is those patients who have SSD because they are able to compare their implanted and nonimplanted sides. Studies have shown that there is both subjective and objective improvement in those patients who are implanted. ${ }^{74,75}$ These improvements include better localization of sound including that of a difficult hearing environment and interaural level differences. $^{75,76}$

In general, CI patients experience significant improvements in both objective and patient-reported measures of QOL. Low rates of failure or nonuse of the device help reassure patients and providers that this technology is worth the effort to improve the patient's hearing and ultimately their quality of life. 
Myth: Patients will have permanent dizziness after $\mathrm{CI}$.

Fact: While transient balance disturbance is possible, permanent disabling vertigo or disequilibrium is rare.

Since CI requires a surgical intrusion into the inner ear, it is natural to expect that the surgery may disturb the inner ear balance system. Some CI recipients do indeed report postoperative vertigo, dizziness, or imbalance. Since the terminology used to describe vestibular symptoms is imprecise and the population of $\mathrm{CI}$ candidates is heterogenous in their baseline vestibular status, the reported incidence of such symptoms over the last two decades has been widely variable. A recent systematic review pooled data from 95 studies to show that on average $9.3 \%$ of patients of all ages suffered from postoperative vertigo after CI, where the term vertigo is loosely defined. ${ }^{77}$ When the data are subdivided by age groups, it appears that older patients are more susceptible to postoperative vertigo. However, this appearance is tempered by the realization that older patients may already have more preoperative vertigo to begin with.

A meta-analysis of 46 eligible studies with matched pre- and postoperative data found that the risk ratio of new vertigo onset after $\mathrm{CI}$ is 2.03 (1.54-2.66). ${ }^{77}$ Furthermore, even though dizziness of different quality and duration has been described after CI, the dizziness uniformly gets better with time, and the incidence of longterm disability due to vestibular dysfunction after unilateral CI is low. ${ }^{78-80}$ The incidence of persistent disabling vertigo at 1 year after surgery in patients who did not have vertigo before surgery is $1.2 \% .^{81}$ An early, prospective observational study of both children and adults undergoing CI showed that the Dizziness Handicap Inventory (DHI) score remains the same or gets better after surgery. ${ }^{81} \mathrm{~A}$ metaanalysis of 27 eligible studies found that $\mathrm{CI}$ has no significant impact on DHI score. ${ }^{82}$

On rare occasions, a patient may suffer chronic debilitating dizziness after CI. Such a case is almost always associated with bilateral severe vestibular hypofunction. It is of great interest, therefore, to determine the effect that CI has on the function of the different vestibular sensors. Traditionally, bithermal caloric irrigation has been the most reported vestibular function test result in connection with $\mathrm{CI}$, but it only measures LF stimulation of the horizontal semicircular canal, approximately equivalent to 0.001 to $0.01 \mathrm{~Hz}$ of head rotation, which has minimal impact on gaze stabilization. ${ }^{80}$

Just like the reported incidence of vestibular symptoms, the reported incidence of reduced caloric response varies widely among individual studies. A meta-analysis of 27 studies in adults demonstrates that CI has a significant negative effect on the results of caloric as well as cervical vestibular evoked myogenic potential (cVEMP) tests. ${ }^{82}$ A different meta-analysis of 46 studies in adults confirmed the negative effect of $\mathrm{CI}$ on caloric irrigation and cVEMP test in finding that the risk ratio of reduced horizontal semicircular canal function is $0.20(0.12-0.34)$, and the risk ratio of impaired cVEMP is 0.10 (0.050.21). ${ }^{77} \mathrm{CI}$ has no significant effect on clinical head impulse test or computerized dynamic posturography. ${ }^{82}$

Another meta-analysis pooled data from 16 studies into $2 \times 2$ tables to calculate the sensitivity and specificity of caloric test, cVEMP, and HIT in detecting vestibular symptom after CI. The analysis of prevalence reveals that in patients with postoperative vestibular symptoms, cVEMP is the most often impaired, the head impulse test is the most often preserved, and no single test has greater than $50 \%$ sensitivity. ${ }^{83}$ That cVEMP is the most often impaired after CI makes sense because the saccule is the closest vestibular sensor to the cochlea and the one most frequently damaged in histological studies of the temporal bone after CI. ${ }^{84,85}$

In a study using quantitative head impulse test with scleral coil, only $3.6 \%$ developed profound vestibular loss, although $31 \%$ demonstrated impaired cVEMP. ${ }^{80}$ In another study, patients older than 70 years had a sevenfold higher risk of sustaining complete vestibular loss in the implanted ear (22 vs. $3 \%$ ) event, though they were no more likely to show vestibular symptoms than younger patients. ${ }^{79}$ The above data would suggest therefore that the chances of unilateral CI causing significant vestibular dysfunction is small, but compounding risk will need to be considered when preexisting vestibular dysfunction is present, 
or when undertaking bilateral CI either concurrent or sequential. In these situations, comprehensive evaluation of all five vestibular sensors can help stratify risks. ${ }^{83}$

Patients with Meniere's disease present a special opportunity and risk in CI. A patient with poor hearing and uncontrolled vertigo in a singularly affected ear may become a candidate for simultaneous labyrinthectomy and CI in the same ear. A patient already status post labyrinthectomy may receive a $\mathrm{CI}$ in the same ear later. And a patient with poor hearing and active vertigo may receive sufficient vestibular deafferentation from a $\mathrm{CI}$ that the vertigo could come under control without labyrinthectomy. In all cases, the patients are likely to achieve hearing benefits from the CI. ${ }^{86,87}$ In patients with bilateral Meniere's disease, however, it would be wiser to implant the poorer balancing ear rather than the poorer hearing ear to avoid deleterious bilateral vestibular hypofunction. ${ }^{86}$

CI can provide great benefits in hearing with transient vestibular symptoms and minor vestibular deficits in some patients. The negative effect on the vestibular system is mostly on the saccule and a little on the LF response of the horizontal semicircular canal. No significant effect of CI has been found on the more physiologically relevant high-frequency response of the semicircular canals, posturography, or DHI. Overall, the clinical effect of CI surgery on the vestibular function is likely to be minimal for most patients. Older adults and patients with preexisting vestibular conditions can safely undergo CI with appropriate preoperative vestibular workup as indicated. MRI.

Myth: Patients with CI cannot undergo

False: Patients with modern CIs can undergo MRI, with a few caveats.

When considering moving forward with $\mathrm{CI}$, patients and their physicians may consider the compatibility of the device with MRI scanning. It is likely that many people will need an MRI at some point in their lifetime. For patients who do undergo an MRI with their $\mathrm{CI}$ in place, there will be image distortion if the MRI is of the head or neck. Overall, the device manufacturers all agree that the area needing the MRI should not be in the artifact zone of the implant itself for optimal visualization. The magnet causes most of the distortion for the MRI image. For patients who need an MRI of the head or neck, removal of the magnet may be necessary to visualize critical structures even in those with MRI-compatible magnets. Additionally, even with all conditions in accordance with manufacturer's recommendations, there can still be risks associated with the scan, including device movement, damage to device, weakening of the magnet, uncomfortable sounds or sensations, implant heating, and image artifact. Additionally, with some manufacturers' models, magnets need to be surgically removed and then replaced to undergo MRI. ${ }^{88-90}$

The key considerations with any implant and MRI include the device itself and the strength of the MRI in Tesla. In the United States, three device manufacturers have FDA approval for CI and MRI safety. As all safety recommendations are subject to change, we strongly recommend that patients and their providers check on the manufacturer's Web site for the most up-to-date information. Additionally, at the time of writing this document, Oticon Medical had newly obtained FDA clearance for its CI in the United States. No information is yet available for this fourth device manufacturer about its MRI safety profile. We have summarized the current MRI compatibility recommendations for the other three U.S. approved manufacturers in Table 1.

Advanced Bionics implants that are currently being implanted in the United States are all MRI conditional. Older models are all MRI contraindicated. A head wrap is not required for any models. ${ }^{89}$ Cochlear nucleus implants that are currently available in the United States are MRI compatible. Newer generations of implants may be MRI compatible, but older models require magnet removal prior to MRI. ${ }^{88}$ MED-EL implants are all MRI conditional. An elastic head wrap is required and the timing of the scan should be at least 6 months postimplantation. ${ }^{90}$

Overall, in most cases, with the correct precautions MRI can be performed safely in CI users. 


\section{CONCLUSION}

Cochlear implantation is a safe, reliable, and well-tolerated rehabilitative option for those who receive limited benefit from traditional hearing aid amplification. This life-changing treatment is a covered benefit for most Americans and the risk of serious or permanent complication related to surgery is low. The audiometric criteria for CI have evolved over time and more patients with residual hearing are now eligible to benefit from CI.

\section{DISCLOSURES}

S.E.M.-Paid consultant for Cochlear Americas for educational content to their Web site, educational consultant to Stryker-reimbursed for travel expenses, paid consultant to OtoMed. Other authors have no disclosures.

\section{REFERENCES}

1. Cochlear implants in adults and children. NIH Consens Statement 1995;13(02):1-30

2. Hodges AV, Schloffman J, Balkany T. Conservation of residual hearing with cochlear implantation. Am J Otol 1997;18(02):179-183

3. Fraysse B, Dillier N, Klenzner T et al. Cochlear implants for adults obtaining marginal benefit from acoustic amplification: a European study. Am J Otol 1998;19(05):591-597

4. Gantz BJ, Turner CW. Combining acoustic and electrical hearing. Laryngoscope 2003;113(10): 1726-1730

5. Gantz BJ, Dunn C, Oleson J, Hansen M, Parkinson A, Turner C. Multicenter clinical trial of the Nucleus Hybrid S8 cochlear implant: final outcomes. Laryngoscope 2016;126(04):962-973

6. Gantz BJ, Dunn CC, Oleson J, Hansen MR. Acoustic plus electric speech processing: longterm results. Laryngoscope 2018;128(02):473-481

7. FDA Access Data. 27.8 Accessed May 31, 2021 at: https://www.accessdata.fda.gov/cdrh_docs/pdf/ p000025s084b.pdf

8. FDA Access Data. 26.7 Accessed May 31, 2021 at: https://www.accessdata.fda.gov/cdrh_docs/pdf13/ p130016a.pdf

9. Turner CW, Reiss LAJ, Gantz BJ. Combined acoustic and electric hearing: preserving residual acoustic hearing. Hear Res 2008;242(1-2):164-171

10. Karsten SA, Turner CW, Brown CJ, Jeon EK, Abbas PJ, Gantz BJ. Optimizing the combination of acoustic and electric hearing in the implanted ear. Ear Hear 2013;34(02):142-150 
11. Tejani VD, Brown CJ. Speech masking release in Hybrid cochlear implant users: roles of spectral and temporal cues in electric-acoustic hearing. J Acoust Soc Am 2020;147(05):3667

12. Woodson EA, Reiss LAJ, Turner CW, Gfeller K, Gantz BJ. The Hybrid cochlear implant: a review. In: Cochlear Implants and Hearing Preservation. KARGER; 2009. Doi: 10.1159/000262604

13. Balkenhol T, Wallhäusser-Franke E, Rotter N, Servais JJ. Cochlear implant and hearing aid: objective measures of binaural benefit. Front Neurosci 2020;14:586119

14. Gifford RH, Dorman MF, Skarzynski H et al. Cochlear implantation with hearing preservation yields significant benefit for speech recognition in complex listening environments. Ear Hear 2013;34 (04):413-425

15. Parkinson AJ, Rubinstein JT, Drennan WR, Dodson C, Nie K. Hybrid music perception outcomes: implications for melody and timbre recognition in cochlear implant recipients. Otol Neurotol 2019;40 (03): $283-\mathrm{e} 289$

16. Gfeller KE, Olszewski C, Turner C, Gantz B, Oleson J. Music perception with cochlear implants and residual hearing. Audiol Neurotol 2006;11(1, Suppl 1):12-15

17. Woodson E, Smeal M, Nelson RC, Haberkamp T, Sydlowski S. Slim perimodiolar arrays are as effective as slim lateral wall arrays for functional hearing preservation after cochlear implantation. Otol Neurotol 2020;41(06):e674-e679

18. Mamelle E, Granger B, Sterkers O et al. Longterm residual hearing in cochlear implanted adult patients who were candidates for electro-acoustic stimulation. Eur Arch Otorhinolaryngol 2020;277 (03):705-713

19. Adunka OF, Gantz BJ, Dunn C, Gurgel RK, Buchman CA. Minimum reporting standards for adult cochlear implantation. Otolaryngol Head Neck Surg 2018;159(02):215-219

20. Spitzer ER, Waltzman SB, Landsberger DM, Friedmann DR. Acceptance and benefits of electro-acoustic stimulation for conventional-length electrode arrays. Audiol Neurotol 2021;26(01):17-26

21. Ludwig S, Riemann N, Hans S et al. Evaluation of hearing preservation in adults with a slim perimodiolar electrode. Eur Arch Otorhinolaryngol 2021; (ePub ahead of print). Doi: 10.1007/s00405-02106755-z

22. Sorkin D. Cochlear implant insurance. Accessed May 25, 2021 at: https://www.acialliance.org/ page/HealthIns

23. Neguse J. Ally's Act - HR 477. US House of Representatives2021. Accessed May 25, 2021 at: https:/www.congress.gov/bill/117th-congress/ house-bill/477

24. Rundshagen I. Postoperative cognitive dysfunction. Deutsches Aerzteblatt Online. Published online February 21, 2014. Doi: 10.3238/ arztebl.2014.0119

25. Coelho DH, Yeh J, Kim JT, Lalwani AK. Cochlear implantation is associated with minimal anesthetic risk in the elderly. Laryngoscope 2009;119(02): 355-358

26. Carlson ML, Breen JT, Gifford RH et al. Cochlear implantation in the octogenarian and nonagenarian. Otol Neurotol 2010;31(08):1343-1349

27. Spitzer ER, Waltzman SB. Outcomes of cochlear implantation in adults over 85 years of age. $\mathrm{Co}^{-}$ chlear Implants Int 2021;22(05):296-302

28. Eshraghi AA, Rodriguez M, Balkany TJ et al. Cochlear implant surgery in patients more than seventy-nine years old. Laryngoscope 2009;119 (06):1180-1183

29. Toner JG, John G, McNaboe EJ. Cochlear implantation under local anaesthesia, the Belfast experience. J Laryngol Otol 1998;112(06):533-536

30. Dietz A, Wüstefeld M, Niskanen M, Löppönen H. Cochlear implant surgery in the elderly: the feasibility of a modified suprameatal approach under local anesthesia. Otol Neurotol 2016;37(05): 487-491

31. Pateron B, Bakhos D, LeLouarn A et al. Local anaesthesia and conscious sedation for cochlear implantation: experience with 20 patients. J Laryngol Otol 2016;130(02):151-156

32. Shabashev S, Fouad Y, Huncke TK, Roland JT. Cochlear implantation under conscious sedation with local anesthesia; safety, efficacy, costs, and satisfaction. Cochlear Implants Int 2017;18(06): 297-303

33. Toner F, Jackson CP, Toner JG. How we do it: local anaesthetic cochlear implantation. Cochlear Implants Int 2013;14(04):232-235

34. Carlson ML. Cochlear implantation in adults. N Engl J Med 2020;382(16):1531-1542

35. Chen DS, Clarrett DM, Li L, Bowditch SP, Niparko JK, Lin FR. Cochlear implantation in older adults: long-term analysis of complications and device survival in a consecutive series. Otol Neurotol 2013;34(07):1272-1277

36. Wong DJY, Moran M, O'Leary SJ. Outcomes after cochlear implantation in the very elderly. Otol Neurotol 2016;37(01):46-51

37. Sorrentino T, Coté M, Eter E et al. Cochlear reimplantations: technical and surgical failures. Acta Otolaryngol 2009;129(04):380-384

38. Balkany TJ, Hodges AV, Buchman CA et al. Cochlear implant soft failures consensus development conference statement. Otol Neurotol 2005;26 (04):815-818

39. Battmer R-D, Linz B, Lenarz T. A review of device failure in more than 23 years of clinical experience of a cochlear implant program with more than 3,400 implantees. Otol Neurotol 2009;30(04): 455-463 
40. Wang JT, Wang AY, Psarros C, Da Cruz M. Rates of revision and device failure in cochlear implant surgery: a 30-year experience. Laryngoscope 2014; 124(10):2393-2399

41. Dutt SN, Ray J, Hadjihannas E, Cooper H, Donaldson I, Proops DW. Medical and surgical complications of the second 100 adult cochlear implant patients in Birmingham. J Laryngol Otol 2005;119(10):759-764

42. Schow B, Friedland DR, Jensen J, Burg L, Runge CL. Electrode failure and device failure in adult cochlear implantation. Cochlear Implants Int 2012; 13(01):35-40

43. Rivas A, Marlowe AL, Chinnici JE, Niparko JK, Francis HW. Revision cochlear implantation surgery in adults: indications and results. Otol Neurotol 2008;29(05):639-648

44. Basavaraj S, Najaraj S, Shanks M, Wardrop P, Allen AA. Short-term versus long-term antibiotic prophylaxis in cochlear implant surgery. Otol Neurotol 2004;25(05):720-722

45. Gawęcki W, Karlik M, Borucki Ł, Szyfter-Harris J, Wróbel M. Skin flap complications after cochlear implantations. Eur Arch Otorhinolaryngol 2016; 273(12):4175-4183

46. Gluth MB, Singh R, Atlas MD. Prevention and management of cochlear implant infections. $\mathrm{Co}^{-}$ chlear Implants Int 2011;12(04):223-227

47. Spitzer JB, Cellum IP, Bosworth C. Stability of audiometric measures and challenges in longterm management of the elderly cochlear implant patient. Otol Neurotol 2013;34(09): 1636-1641

48. Brito R, Monteiro TA, Leal AF, Tsuji RK, Pinna $\mathrm{MH}$, Bento RF. Surgical complications in 550 consecutive cochlear implantation. Rev Bras Otorrinolaringol (Engl Ed) 2012;78(03):80-85

49. Reefhuis J, Honein MA, Whitney CG et al. Risk of bacterial meningitis in children with cochlear implants. N Engl J Med 2003;349(05):435-445

50. Centers for Disease Control and Prevention. Cochlear implants and vaccination recommendations. Published August 8, 2019. Accessed June 19, 2021 at: https://www-cdc-gov.laneproxy.stanford.edu/ vaccines/vpd/mening/public/dis-cochlear-faq-gen. html

51. Wei BPC, Robins-Browne RM, Shepherd RK, Clark GM, O'Leary SJ. Can we prevent cochlear implant recipients from developing pneumococcal meningitis? Clin Infect Dis 2008;46(01):e1-e7

52. Torkos A, Czigner J, Jarabin J et al. Recurrent bacterial meningitis after cochlear implantation in a patient with a newly described labyrinthine malformation. Int J Pediatr Otorhinolaryngol 2009;73 (01):163-171

53. Yoshikawa N, Hirsch B, Telischi FF. Cochlear implant fixation and dura exposure. Otol Neurotol 2010;31(08):1211-1214
54. Cohen NL, Roland JT Jr, Marrinan M. Meningitis in cochlear implant recipients: the North American experience. Otol Neurotol 2004;25(03):275-281

55. Fayad JN, Wanna GB, Micheletto JN, Parisier SC. Facial nerve paralysis following cochlear implant surgery. Laryngoscope 2003;113(08):1344-1346

56. Jeppesen J, Faber CE. Surgical complications following cochlear implantation in adults based on a proposed reporting consensus. Acta Otolaryngol 2013;133(10):1012-1021

57. Halawani R, Aldhafeeri A, Alajlan S, Alzhrani F. Complications of post-cochlear implantation in 1027 adults and children. Ann Saudi Med 2019; 39(02):77-81

58. Alzhrani F, Lenarz T, Teschner M. Taste sensation following cochlear implantation surgery. Cochlear Implants Int 2013;14(04):200-206

59. Niparko JK, Oviatt DL, Coker NJ, Sutton L, Waltzman SB, Cohen NLVA Cooperative Study Group on Cochlear Implantation. Facial nerve stimulation with cochlear implantation. Otolaryngol Head Neck Surg 1991;104(06):826-830

60. Cohen NL, Hoffman RA, Stroschein M. Medical or surgical complications related to the nucleus multichannel cochlear implant. Ann Otol Rhinol Laryngol 1989;98(09):754

61. Alzhrani F, Halawani R, Basodan S, Hudeib R. Investigating facial nerve stimulation after cochlear implantation in adult and pediatric recipients. Laryngoscope 2021;131(02):374-379

62. Mueller CA, Khatib S, Temmel AFP, Baumgartner W-D, Hummel T. Effects of cochlear implantation on gustatory function. Ann Otol Rhinol Laryngol 2007;116(07):498-501

63. Baguley DM, Atlas MD. Cochlear implants and tinnitus. Prog Brain Res 2007;166:347-355

64. Ito J. Tinnitus suppression in cochlear implant patients. Otolaryngol Head Neck Surg 1997;117 (06):701-703

65. Kloostra FJJ, Verbist J, Hofman R, Free RH, Arnold R, van Dijk P. A prospective study of the effect of cochlear implantation on tinnitus. Audiol Neurotol 2018;23(06):356-363

66. Kompis M, Pelizzone M, Dillier N, Allum J, DeMin N, Senn P. Tinnitus before and 6 months after cochlear implantation. Audiol Neurotol 2012; 17(03):161-168

67. Baguley D. New insights into tinnitus in cochlear implant recipients. Cochlear Implants Int 2010; 11:3136

68. McRackan TR, Bauschard M, Hatch JL et al. Meta-analysis of quality-of-life improvement after cochlear implantation and associations with speech recognition abilities. Laryngoscope 2018;128(04): 982-990

69. McRackan TR, Hand BN, Velozo CA, Dubno JRCochlear Implant Quality of Life Development Consortium. Cochlear implant quality of life 
(CIQOL): development of a profile instrument (CIQOL-35 Profile) and a global measure (CIQOL-10 Global). J Speech Lang Hear Res 2019;62(09):3554-3563

70. McRackan TR, Hand BN, Velozo CA, Dubno JRCochlear Implant Quality of Life Consortium. Validity and reliability of the Cochlear Implant Quality of Life (CIQOL)-35 Profile and CIQOL10 Global instruments in comparison to legacy instruments. Ear Hear 2021;42(04):896-908

71. Eapen RJ, Buss E, Adunka MC, Pillsbury HC III, Buchman CA. Hearing-in-noise benefits after bilateral simultaneous cochlear implantation continue to improve 4 years after implantation. Otol Neurotol 2009;30(02):153-159

72. Laszig R, Aschendorff A, Stecker M et al. Benefits of bilateral electrical stimulation with the nucleus cochlear implant in adults: 6-month postoperative results. Otol Neurotol 2004;25(06):958-968

73. Lane C, Zimmerman K, Agrawal S, Parnes L. Cochlear implant failures and reimplantation: a 30year analysis and literature review. Laryngoscope 2020;130(03):782-789

74. Dorman MF, Natale SC, Butts AM, Zeitler DM, Carlson ML. The sound quality of cochlear implants: studies with single-sided deaf patients. Otol Neurotol 2017;38(08):e268-e273

75. Prejban DA, Hamzavi J-S, Arnoldner C et al. Single sided deaf cochlear implant users in the difficult listening situation: speech perception and subjective benefit. Otol Neurotol 2018;39(09): e803-e809

76. Dorman MF, Cook Natale S, Baxter L, Zeitler DM, Carlson ML, Noble JH. Cochlear place of stimulation is one determinant of cochlear implant sound quality. Audiol Neurotol 2019;24(05): 264-269

77. Hänsel T, Gauger U, Bernhard N et al. Metaanalysis of subjective complaints of vertigo and vestibular tests after cochlear implantation. Laryngoscope 2018;128(09):2110-2123

78. Kubo T, Yamamoto K, Iwaki T, Doi K, Tamura M. Different forms of dizziness occurring after cochlear implant. Eur Arch Otorhinolaryngol 2001;258 (01):9-12
79. Enticott JC, Tari S, Koh SM, Dowell RC, O'Leary SJ. Cochlear implant and vestibular function. Otol Neurotol 2006;27(06):824-830

80. Melvin T-AN, Della Santina CC, Carey JP, Migliaccio AA. The effects of cochlear implantation on vestibular function. Otol Neurotol 2009;30(01):87-94

81. Buchman CA, Joy J, Hodges A, Telischi FF, Balkany TJ. Vestibular effects of cochlear implantation. Laryngoscope 2004;114(10 Pt 2, Suppl 103):1-22

82. Ibrahim I, da Silva SD, Segal B, Zeitouni A. Effect of cochlear implant surgery on vestibular function: meta-analysis study. J Otolaryngol Head Neck Surg 2017;46(01):44

83. Abouzayd M, Smith PF, Moreau S, Hitier M. What vestibular tests to choose in symptomatic patients after a cochlear implant? A systematic review and meta-analysis. Eur Arch Otorhinolaryngol 2017;274(01):53-63

84. Tien H-C, Linthicum FH Jr. Histopathologic changes in the vestibule after cochlear implantation. Otolaryngol Head Neck Surg 2002;127(04):260-264

85. Handzel O, Burgess BJ, Nadol JB Jr. Histopathology of the peripheral vestibular system after cochlear implantation in the human. Otol Neurotol 2006; 27(01):57-64

86. Mukherjee P, Eykamp K, Brown D et al. Cochlear implantation in Ménière's disease with and without labyrinthectomy. Otol Neurotol 2017;38(02): 192-198

87. Selleck A, Dillon M, Perkins E, Brown K. Cochlear implantation in the setting of Meniere's disease after labyrinthectomy: a meta-analysis. Otol Neurotol 2021;42(08):e973-e979

88. Cochlear Americas. MRI guidelines for professionals Accessed June 19, 2021 at: https://www.cochlear.com/us/en/professionals/resources-and-training/ mri-guidelines

89. Advanced Bionics LLC. MRI Safety Information Accessed May 8, 2021 at: https://advancedbionics. $\mathrm{com} / \mathrm{us} / \mathrm{en} /$ portals/professional-portal/products/ mri-safety.html

90. MED-EL Corporation. Important Safety Information Accessed May 8, 2021 at: https://www. medel.com/en-us/important-safety-information 